\title{
PRODUCCIÓN DE MATERIA SECA Y CONCENTRACIÓN DE FÓSFORO FOLIAR DE FORRAJERAS HERBÁCEAS NATIVAS CON FERTILIZACIÓN FOSFATADA
}

\author{
Porta, Miriam; Hack, C.M.; Tomei, C.E. \\ Instituto Agrotécnico "Pedro M. Fuentes Godo", Facultad de Ciencias Agrarias, UNNE
}

Los campos naturales constituyen el principal recurso forrajero, en cuanto a superficie, para la ganadería de la región del nordeste argentino (NEA) (Tomei, 1995). El fósforo (P) es uno de los nutrientes deficitarios encontrados con mayor frecuencia en los suelos de esta región. Este elemento es esencial para el normal crecimiento y desarrollo de las pasturas perennes, en especial en los estadios iniciales del desarrollo de las plantas. La corrección temprana de su deficiencia mejora significativamente la implantación de las pasturas, fundamentalmente del componente leguminoso (Fernández et. al, 1993).

La deficiencia de $\mathrm{P}$ provoca una marcada reducción del crecimiento y la calidad del forraje, su disponibilidad juega un rol fundamental en el sistema suelo: planta: animal definiendo la productividad del sistema.

Varios ensayos de fertilización tanto a nivel de parcelas como con animales han demostrado que la aplicación de fertilizantes fosfóricos incrementa la producción de materia seca (MS) entre un 30 y un $40 \%$, mejora substancialmente el contenido de leguminosas nativas y aumenta en un $60 \%$ el contenido de $P$ en el pasto (Pizzio, 1995).

El agregado de fertilizantes fosfatados en suelos de la Provincia de Corrientes produjo cambios significativos en el contenido de $P$ foliar en pastizales naturales, alcanzando valores máximos con el agregado de $90 \mathrm{~kg} \mathrm{P} \mathrm{ha}^{-1}$ (Porta et al., 2006).

El objetivo de este trabajo fue estudiar la respuesta de forrajeras herbáceas nativas al agregado de dosis crecientes de $\mathrm{P}$ en base a la producción de MS y contenido de fósforo foliar.

El trabajo se efectuó bajo invernáculo en la Facultad de Ciencias Agrarias. Se tomaron muestras de suelos provenientes de dos localidades de la Provincia de Corrientes (Argentina), un Argiudol vértico de Empedrado y un Kandihumult típico de Gobernador Virasoro. Las muestras se extrajeron en forma de panes de suelo de $20 \mathrm{~cm}$ de diámetro y $15 \mathrm{~cm}$ de profundidad, con su correspondiente cubierta vegetal, luego fueron acondicionadas en macetas y se mantuvieron bajo condiciones controladas de riego.

En cada suelo se probaron cuatro tratamientos: P0 sin el agregado de fertilizante, $\mathrm{P} 1$ con $30, \mathrm{P} 2$ con 60 y $\mathrm{P} 3$ con $90 \mathrm{~kg} \mathrm{P}$. ha ${ }^{-1}$. El $\mathrm{P}$ fue suministrado como superfosfato triple de calcio. Se emplé un diseño completamente aleatorizado con tres repeticiones.

Al inicio del ensayo se realizó un corte de emparejamiento, luego se realizaron seis cortes de la cubierta vegetal durante la estación de crecimiento cuando las plantas alcanzaron una altura de $15-20$ $\mathrm{cm}$, entre enero y septiembre, cortando con tijera a un altura de $2,5 \mathrm{~cm}$. El material cosechado fue llevado a estufa de tiro forzado a $60^{\circ} \mathrm{C}$ hasta peso constante, para determinar MS. Una vez pesado el material vegetal fue molido y se realizo una digestión nitrica perclórica, para determinar el $P$ asimilado (PA) por las plantas con el método colorimétrico de Murphy-Riley. Los datos obtenidos se analizaron con varianza y las diferencias entre medias se probaron con el Test de Tukey $(\mathrm{p}<0,05)$.

\section{Fósforo Asimilado:}

El agregado de fertilizantes fosfatados en ambos suelos produjo cambios significativos en el contenido de PA (Figura 1).

En la vegetación de Virasoro todas las dosis de fertilizantes produjeron un incremento significativo

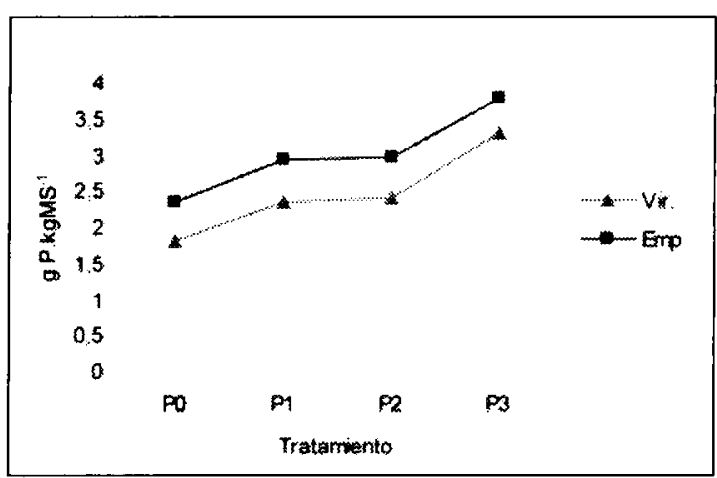

Figura 1. Contenido de PA por tratamiento para ambas localidades. 
Tabla 2: Producción de MS correspondientes a la vegetación de las localidades de Gobernador Virasoro y Empedrado.

\begin{tabular}{ccc}
\hline Tratamientos & \multicolumn{2}{c}{ MS } \\
\hline P0 & Virasoro & Empedrado \\
\hline P1 & $4,65 \mathrm{~A}$ & $3,13 \mathrm{~A}$ \\
P2 & $4,85 \mathrm{AB}$ & $5,13 \mathrm{~B}$ \\
P3 & $6,42 \mathrm{~B}$ & $4,23 \mathrm{AB}$ \\
CV & $4,81 \mathrm{AB}$ & $4,77 \mathrm{~B}$ \\
DMS & 42,47 & 47,42 \\
\hline
\end{tabular}

*Letras distintas en una columna indican diferencias significativas $(\mathrm{p}<=0,05)$

en el contenido de PA con respecto al testigo. El tratamiento $\mathrm{P} 3$ tuvo un $83 \%$ más de $\mathrm{P}$ por $\mathrm{kg}$ de MS que P0 y difirió significativamente de Pl y P2. En las macetas de la localidad de Empedrado P3 también respondió favorablemente a la fertilización. La dosis máxima de $\mathrm{P}$ produjo un incremento del $60 \%$ en PA respecto a P0. Las curvas de respuesta a la fertilización fosfatada, tienen la misma tendencia en la vegetación de ambos suelos, siendo siempre el tratamiento P3 el que determino mayores respuestas.

\section{Materia Seca:}

La producción de MS se vio incrementada por el agregado de $\mathrm{P}$ en ambos suelos (Tabla 2 ).

En la vegetación correspondiente a la localidad de Virasoro, el tratamiento $\mathrm{P} 2$ rindió un $38 \%$ más que P0, difiriendo significativamente del mismo. Los tratamientos $\mathrm{Pl}$ y $\mathrm{P} 3$, con valores intermedios de producción, no manifestaron diferencias respecto a los demás tratamientos.

En cuanto a la respuesta de la vegetación en el suelo de Empedrado, el agregado de la dosis más baja de $\mathrm{P}$ provocó la mayor diferencia con respecto a testigo, siendo el rendimiento de P1 un 64\% superior a P0 (Tabla 2).

El agregado de $30 \mathrm{~kg} \mathrm{P.} \mathrm{ha-}{ }^{-1}$ ya sería suficiente para incrementar la producción de MS, en cuanto al PA por las plantas.

La mayor dosis de $\mathrm{P}$ (P3) produjo los valores máximos de PA en ambos suelos.

\section{BIBLIOGRAFÍA:}

Fernández, G F; Benítez, C A; Royo Pallarés, O.; Pizzio, R 1993. Principales forrajes nativas del medio de la provincia de Corrientes. Serie técnica $\mathrm{N}^{\circ} 232^{\circ}$ edición. INTA-EEA Mercedes, Corrientes, Arg. 91 pp.

Pizzio, R M. 1995. Utilización y manejo de los pastizales del ecosistema Campos de Argentina IICA-BJD-PROCISUR Dialogo XL-Utilización y manejo de pastizales. 115-126.

Porta, M.; Hack, C.M.; Tomei, C.E 2006. Fertilización fosfatada sobre pastizales de la provincia de Corrientes. Ensayo en macetas. Reunión de comunicaciones cientificas y tecnológicas. Secretaría General de Ciencia y Técnica, UNNE, realizado en Resistencia- Chaco.

Tomei, C.E. 1995. Manejo racional de los campos naturales para mejorar la producción ganadera en la región chaqueña. Ganadería subtropical. Jornadas internacionales de actualización. 6166 pp. 